\title{
XIII.
}

\section{Ueber ein charakteristisches Symptom der eitrigen Thrombose des sinus longitudinalis superior. Von}

Prof. G. Gradenigo in Turin.

Das Symptom einer eiterigen Thrombose des sinus longit sup., auf welches ich hier die Aufmerksamkeit lenken möchte, besteht in dem Auftreten einer fluktuierenden schmerzhaften Sehwellung in der Seheitelgegend und zwar in der Mittellinie, entsprechend einem der foramina emissaria Santorini, also im hinteren Teile der sutura sagittalis. Die Sehwellung kann einfach Blut oder auch Eiter mit Granulationen enthalten.

Die eiterige Thrombose des sinus longitudinalis otogenen Ursprungs wird bei Autopsien ziemlich selten angetroffen, und zwar in jenen Fällen von otogener Thrombose des sinus sigmoideus, in welchen der Thrombus sich auf den Confluens sinuum erstreckt.

Zuweilen ist die eiterige Thrombose auf den dem Eingange zum Confluens sinuum zunächst gelegenen Absehnitt des sinus longitudinalis beschränkt, andere Male dehnt sie sich auf die hintere Hälfte des sinus longitudinalis aus. Auf jeden Fall ist die Thrombose nur partial und verursacht gewöhnlich keine sehon im Leben erkennbaren Symptome.

Hessler hat in seinem Werke iiber die otogene Pyämie $\left.{ }^{1}\right)$ in welchem er alle bis zum Jahre 1895 bekannt gewordenen Beobachtungen von otogener eiteriger Thrombose zu sammeln versuchte 11 Fälle (auf 398 Beobachtungen) von eiteriger Thrombose des sinus longitudinalis konstatieren können. Bei Kontrollierung dieser Angabe müssen jedoeh 4 von diesen Fällen ausgeschlossen werden, in welchen nämlich der im sinus longitudinalis bei der Antopsie

1) Die otogene Pyämie, Jena (Fischer) 1896. 
vorgefundene Thrombus nicht infektiöser Natur war, auberdem muß von den $T$ übrig bleibenden Fällen auch der von Aberorombie eliminiert werden, weil nach Angabe dieses Autors der Thrombus des sinus lateralis sinister bloß den Anfang des oberen Blutleiters erreichte. Es bleiben demnach nur die folgenden 6 Beobachtungen, in welchen, wie es scheint, intra vitam, gar kein eine Läsion des sinus longitudinalis charakterisierendes Symptom konstatiert werden konnte.

W eil. (De l'inffammation des Sinus cérébraux, 1856). 22 Jabre alter Mann. Chronischej eiterige Mittelohrentzündung auf der rechten Seite. Eiteriger Thrombus im rechten lateralen und im oberen longitudinalen Blutleiter. Caries.

F. Allport (Purulent Brains deposits and Pblebitis, a. Thrombosis of the cerebral veins and sinus, following ear disease. 1892.)

I. Fall. 23 Jahr alter Mann. Acute eiterige rechtsseitige Otitis. Keine Schwellung, nur Schmerzhaftigkeit in der Warzenfortsatzgegend. Die operative Erö́ffnung des Antrum ließ keinen Eiter erkennen. Bei der Autopsie wurde Thrombose des sinus longitudinalis und des sinus petrosus superior, rechts, Abszess im Kleinhirn and Meningitis nachgewiesen. Es schien anch eiterige Thrombose des rechteu sinus lateralis hestanden zu haben, weil nach dem kurzen Berichte, den Hessler über den Fall gab, Caries an der hinteren Seite der Warzengegend (sinus sigmoideus?) vorhanden gewesen ist.

II. Fall. Allport. Ibidem.

Frau, mit eiteriger acuter rechtsseitiger Otitis. Eiterige Thrombose des sinus transversus und longitudinalis. Caries des sulcus sigmoideus. Leptomeningitis.

Kn app (Zeitschr. f. Ohrenheilk. XXIY, p. 161).

25 Jahr alte Frau, eiterige acnte Otitis anf der rechten Seite. Entzündung des Warzenfortsatzes. Kopfweh, Entzündung der Sehnervenpapille. Aphasie, Somnolenz, kein Fieber. Bei der Autopsie wurde ein vereiterter Thrombus im oberen Teile des sinus sigmoideus nachgewiesen, der sich in den sinus longitudinalis und in den linken lateralen Sinus fortsetyte. Thrombose der vena jugularis interna, gleichfalls links. AbsceB im Gehirne und im Kleinhirne.

Jansen. Archiv für Obrenheilkunde. XXXV.p. 285

26 Jahr alter Mann. Rechtsseitige eiterige acute Mittelohrentzündung. Nystagmus, Pupille rechts enger als links. Entzündung der Sehnervenpapille. Kopfweb, spinale Schmerzen, extra duraler Abscess um den Sinus herum. Eiterig zerfallener Thrombus im hinteren Teile des sinus longitudinalis superior, der sich rechts in den sinus lateralis bis $2 \mathrm{~cm}$ weit zum foramen jugulare hin erstreckt. In den linksseitigen sinus lateralis reicht die Eiterung nicht hinein; es war derselbe gegen den sagittalen Blutleiter durch einen festen etwa $1 \mathrm{~cm}$ langen Thrombus geschlossen. Leptomeningitis..

Den vou Hessler zitierten Fällen sind noch die folgenden hinzuzuftugen:

De Carli. I. Fall. (Arebivio Ital di Otologia e Laringologia. XV. p. 449).

23 Jahr alter Mann. Rechtsseitige eiterige chronische Mittelobrentzündung. Wäbrend des Militärdienstes litt P. an Malariafieber. Seit 20 Tagen war Fieber mit pyämischem Charakter vorhanden, auferdem intensiver Kopfschmerz. Die Warzengegend bot nicbts abnormes dar, das Trommelfell war zerstört, die Schleimbaut der Paukenhöhle gerötet. Eiterung feblte. Entzündung der Sehnervenpapille. Steifheit des Nackens. Extraduraler Absceß um den sinus sigmoideus berum. Eiterige Thromhose des sinus lateralis, der eröffinet wurde. Onterbindung der jugularis interna, die leer war. Fortbestehen des Fiehers von pyämischem Charakter. Tod. 
Bei der Autopsie sah man eiterige Thrombose des sinus lateralis auf der rechten Seite, die sich auf den confluens sinuum und auf den sinus longitudinalis superior bis auf eine gewibe nicht näher angegebene Strecke fortsetzte.

Der sinus lateralis sinister war normal.

Intra vitam konnte gar keine abnorme Dilatation der GefäBe des bebaarten Kopfes erkannt werden.

H öl s c ber (Archiv für Ohrenheilkunde. Bd. 52. p. 110).

21 Jahr alter Mann. Rechtsseitige chronische Mittelohrentzündung. P. zeigte die Symptome einer eiterigen Thrombose des rechtsseitigen sinus lateralis und wurde successiv 5 mal operiert, bei Chloroform - oder Äthernarkose. Es war ein eiteriger Thrombus unterbalb des foramen mastoideum vorhanden. Die jugularis interna war an der Stelle der Ligatur gesund. Bei der Autopsie sah man, daß die eiterige Thrombose weit ausgebreitet war, und zwar bis in den confluens sinuum, in den sinus longitudinalis superior und in die Venen der convexen Seite des Gehirnes usw.

Anch ich habe einen Fall von eiteriger Thrombose beobachtet, die sich auf den hinteren Teil des sinus longitudinalis ausdehnte.

C. Agostino, $49 \mathrm{Jahr}$ alt. Es scheint, daß die eiterige Thrombose anfangs auf der oberen Bulbus der rechtsseitigen jugularis beschränkt war. Es konnte intra vitam die Paralyse der rechtsseitigen chorda vocalis und des m. trapezius und sterno-cleido mastoideus, ferner eine Parese der gleichseitigen Hälfte des weichen Gaumens erkannt werden. Bei der Autopsie fand man eiterige Thrombose des rechtsseitigen sinus lateralis, die sich eine Strecke weit in den sinus longitudinalis fortsetzte. Klinische Symptome einer Ausdehnung des Thrombus in den sinus longitudinalis sup. sind nie vorhanden.

Neben diesen Beobachtungen sind in der Literatur noch andere verzeichnet, in welchen bestimmte Symptome darauf zu deuten scheinen, daß eine eiterige Thrombose des sinus longitudinalis sup. vorhanden war.

Sehr kompliziert ist der folgende Fall von $\mathrm{Richter}$, weil gleichzeitig mehrere Sinus von der Thrombose befallen waren und deshalb das vorhanden gewesene Symptom (ödematöse Schwellung des Gesichtes und des Halses) nicht einig und allein auf eine Thrombose des sinus longitudinalis sup. bezogen werden konnte.

Richter (Berl. klinische Wochenschr. 1884. No. 6).

$62 \mathrm{Jahr}$ alte Frau. Rechtsseitige chronische eiterige Mittelrohrentzündung. Parese des $n$ facialis auf der rechten Seite. Erbrechen. Sehwellung der Augenlider, des Gesichtes, des Halses (der behaarten Kopfhaut?) Myosis, Kopfweh, Bopor. Eiterige Thrombose der hinteren Hälfte des sinus longitndinalis und der sinus transversi. Die beiden venae jugulares waren frei. Der sinus cavernosus and die rechtsseitige vena ophtalnalica wareu thrombosiert.

Lermoyez beobachtete einmal die Dilatation der Venen behaarten Kopfhaut und er hält diese Erscheinung als charaktestisch für eine eiterige Thrombose des sinus longitudinalis.

Lermoyez, (Annales des maladies de l'oreille, du nez et du larynx, 1897, p. 497 ).

30 Jahr alte Frau. Rechtsseitige chronische Otorthöe, extraduraler Abscess, eiternde Thrombophlebitis des sinus lateralis rechts, des oberen 
Bulbus und der Vena jugularis interna, des sinus lateralis links, des sinus rectus und des sinus longitudinalis superior. Dieser letztere Sinus war bis zar Einmündung der großen Venen von Trolard thrombosiert. Der vordere Abschnitt desselben war normal. Normal waren aucb der sinus petrosus superior und inferior auf der rechten Seite. Es war bei P. außerdem eine beträchtliche Erweiterung der Venen der behaarten Kopfhaut vorhanden, die in eminenter Weise sich kundgab nach der Entfernung der Haare, welche zur Vorbereitung eines operativen Eingriffes vorgenommen wurde. Die Erweiterung der Venen schreibt L ermo yez der Stase in der collateralen Zirkulation des Venenblutes za, welche im Gefolge der Undurchgăngigkeit des sinus longitudinalis superior sich ausbildete.

Der von mir beobachtete Fall steht, soviel mir bekannt, isoliert in der Literatur, obwohl er Analogien mit einer von De Carli gemachten Beobachtıng (und zwar der zweiten von ihm publizierten) aufweist.

Rechtsseitige acute Mittelohrentzindung bei einer schwangeren Frau. Mastoiditis m it subperiostalem Abscesse. Schmerzhafte Schwellungdes Vorderk opfes, Ödem der Stirngegend und der Lider. Eiterige Thrombose des rechten lateralen und des sinus longitudinalis superior. Leptomeningitis. Tod.

O. Maria, 31 Jahre alte Bäuerin. Die Anamnese ließ bezüglich der Vergangenheit der Kranken nichts Besonderes ermitteln, abgesehen von dem malarischen Fieber, an welchem sie in ihrem 25. Jahre während der Arbeiten an Reisfeldern litt. Patientin heiratete in ihrem 27. Jahre. Drei Monate nach der Verheiratung abortierte sie; spăter, d. h. ungefähr ein Jahr nach der MiBgeburt gebar sie ein Kind, das gesund und stark lebt. Sie hatte dann nochmals eine MiBgeburt, und im April 1904, ein anderes Kind, das auch gegenwärtig noch gesund ist. Ungefähr 15 Tage vor der letzten Geburt, d. h. im Beginne des Monats April 1904 trat bei Patientin eine rechtsseitige akute Mittelohrentzandung mit heftigen Schmerzen am Ohre und auf der rechten Seite des Kopfes, ohne Eiterung im Gehörgange auf. Bald entstand eine schmerzhafte Schwellung, der entsprechend die Haut gerötet war, hinter dem rechten Ohre, welche nach der Beschreibung der Patientin wahrscheinlich durch einen subperiostalen AbszeB des Warzenfortsatzes verursacht war; es wurde derselbe nicht eröffnet und ist blof in verschiedener Weise, hauptsächlich mit Salben, lokal behandelt worden. Der Abszeß eröffnete sich später spontan nach außen, und es bildete sich eine fistulöse Öffnung, welche noch zur Zeit des Eintretens der Patientin in die Klinik bestand. Von derseiben wird noch im Folgenden die Rede sein. Nach Entleerung der Eiteransammlung; welche hinter dem Warzenfortsatze vorhanden war, hörten die Schmerzen auf. Kurze Zeit nachher entstand eine namentlich bei Drack schmerzhafte, deutlich fluktuierende Schwellung, die aber auch der Sitzeiner kontinuierlichen drückenden Schmerzempfindung war, in der Scheitelgegend. Nach und nach traten intensive Kopfschmerzen auf, wegen welcher Patientin sich entschloß, unsere Klinik aufzusuchen.

Status praesens beim Eintritte in unsere Klinik (6. Juli 1904):

Patientin, schlecht genährt, blaß, bietet den Anblick einer sehwer kranken Person Wir richteten unsere Aufmerksamkeit namentlich auf zwei Umstănde: das Leiden am rechten Ohre und die Schwellung in der Scheitelgegend.

Nach unten und etwas nach vorn von der-Spitze des Warzenfortsatzes, rechts, . war die Hant gerötet, und aus einer kleinen, von Granulationen umgeben Offnung kam bei geringem Drucke breiiger Eiter hervor. Bei der Sondierung konnte man erkennen, daB der Fistelgang nach oben und etwas nach hinten sich ungefähr $3 \mathrm{~cm}$ weit erstreckte, daß aber nirgends Knochen entblößt war. Der Gehörgang zeigte sich weit und enthielt keinen Eiter, das Trommelfell nicht perforiert, aber glanzlos. Die Hörschärfe war normal. 
Nasen- und Racbenhöhlenbefund negativ, ebenso der des linken Ohres. Die Zunge dagegen zeigte ein gesprenkeltes Aussehen (Lingua geographica). Syphilitische Alterationen fehlten an der Schleimhaut der Wangen, des Rachens und an der Körperhaut. Am Halse fühlte man auf beiden Seiten vergrößerte Lymphdrüsen; ein kleines Ganglion war auch links in der Gegend der Epitrochlea vorhanden.

Die Schwellung am Scheitel sab in der Nähe der Medianlinie, etwas mehr nach rechts, entsprechend dem binteren Teile der Sutura parietalis; sie hatte die Größe einer halben $\mathrm{NuB}$, war rundlich und von unveränderter Haut bedeckt, weich und fluktuierend, āußerst schmerzhaft bei Berührung und anch spontan, so daß die Kranke in der Nacht nicht schlafen konnte.

Wir dachten zuerst an ein erweichtes Gumma der Seitenwandbeine wegen der zweimaligen Mißgeburten, des Vorhandenseins der vergrößerten Lymphdrüsen an den genannten Stellen, der Persistenz von heftigen Kopfschmerzen, namentlich nachts und wegen des negativen Resultats der Anwendung der gewöhnlichen antineuralgischen Mittel.

Vielleicht bätte die Kranke, wenn man die Diagnose sofort auf Thrombose des Sinus longitudinalis super. gestellt hätte, durch einen prompten operativen Eingriff gerettet werden können.

In dem Zweifel, daß es sich um Syphilis handeln könnte, gaben wir große Dosen von Jodkali mehrere Tage hindurch; es wurde dasselbe vollkommen gut vertragen, batte aber keinen wesentlichen Einflub auf die Schmerzen. Die Schwellung am Scheitel nahm unterdessen zu und umgab sich mit einem ödematösen Hofe. Das Ödem breitete sich rasch auf die Stirngegend und auf die Lider beider Augen aus, was wegen des reichen Haarwuchses nicht sofort wahrgenommen werden konnte. Die Pupillen waren jedoch normal, und es bestanden keine Lasionen am Augenhintergrunde; auch das zentrale and periphere Nervensystem zeigte gar keine Alterationen.

Gleicbzeitig mit der Verschlimmerung der Symptome am Kopfe hörte der Ausfluß des Eiters aus dem Fistelgange am Warzenfortsatze auf und die Fistel schloh sich bald spontan vollständig, obwohl wir sie offen zu erhalten uns anstrebten.

Die Temperatur, welche in den ersten Tagen abends $37,5-37,8^{\circ}$ betrug, stieg (in der Acbselhöhle) auf $38^{\circ} \mathrm{am} 10 . \mathrm{Juli}$.

Es wurde die Verabreichung von Jodkall eingestellt und ein operativer Eingriff beschlossen, der auch am 11. Juli ansgefuhrt worden ist.

Morphin-Chloroformnarkose. Es wurde an der Stelle der Schwellung am Scheitel ein Kreuzschnitt gemacht. Man traf Eiter und Granulationen an; den Grund des Sackes bildete die Galea aponeurotica und war mit Granulationen bedeckt, welche sorgfältigst ausgekratzt worden sind. Bei der Sondenuntersuchung konnte kein entblöBter Knochen nachgewiesen werden. Tamponierung. Die Operation wurde ganz gut vertragen; sie modificierte jedoch nicht im mindesten die Schmerzen im Kopfe, welche wie früher andauerten, namentlich an der Operationsstelle. Während der folgenden Nacbbehandlung konnte bei genauer Untersuchung des Grundes der Operationsstelle Eiter erkannt werden, welche aus einer ganz kleinen Öffnung in der Galea aponeurotica hervorquoll. Mittelst der Sonde, welche nur schwer vordrang, konstatierte man das Vorbandensein von eariösem Knochen.

II. Operation (19). Juli).

Morphin-Chloroformnarkose: Nach Divarikation und Fixierung mit $\mathrm{Ha}-$ ken der von Weichteilen bestehenden Lappen, welche bei der ersten Operation gebildet worden sind, schnitten wir das Periost ein und hoben es in die Höhe. Hierdurch wurde ein Knochenabschnitt von der Größe eines Zweifrankstückes blolgelegt, in der Mittellinie, etwas nach rechts, im hinteren Teile der Sutura sagittalis, der mit schwärzlichen Granulationen bedeckt war. Der Knochen war cariös und die Sonde penetrierte an der hinteren Peripherie und etwas rechts von der bloßgelegten Zone, durch eine Fistelöfinung bindurch, in die Schädelhöhle. Mittelst des Hohlmeißel und Knochen* zange entfernten wir den erkrankten Knochen; der untenliegende Teil der Dura mater war cirkulär, entsprechend der Größe des erkrankten Knochenteiles, granulierend. Zentral in der Dura mater gewahrte man eine öffnung, 
durch welche hindurch man mit der Sonde von links nach rechts und ein wenig von hinten nach vorn vordringen konnte. Bei der Sondierung trat reichlicher breiiger Eiter hervor. Längs der gefurchten Sonde wurde die verdickte Dura mater eingeschnitten und man erkannte, daß die Fistelöffnung in den blutleeren sinus longitudinalis superior fuhrte. Dieser war von einem blutroten Thrombus eingenommen, den wir nicht entfernten, hauptsächlich weil die Kranke keine pyämischen symptome anfwies. Hingegen wurde mit der Scheere von der äußeren Wand des sinus ein großer Teil entfernt und schließlich tamponiert.

Die Narkose verlief während der Operation ganz gut, am Schlusse derselben jedoch erbrach die Kranke eine grinliche Flussigkeit, welche zum Teil in die Atmungswege zurückflok, so daß die Atmung oberlächlich und auch der Puls kleiner wurde. Nachdem aber Mund- und Rachenhöble mit Gazetamponen wiederbolt gereinigt, die Zunge rhythmisch hervorgezogen und zwei Injelktionen mit Coffeïn gemacht worden sind, erholte sich die Kranke und es zeigten sich auch successiv gar keine Symptome von Seite der Bronchien oder der Lungen.

In den folgenden Tagen verschlimmerte sich der Zustand der Kranken; die Kopfschmerzen wurden immer intensiver, die Temperatur war fast konstant $39,5-40^{\circ}$, und es traten deutliche Symptome von Meningitis auf (Steifheit des Nackens und der Wirbelsäule, Kernigsches Symptom, Herzschwäche, Trägbeit der Pupillenreaktion, und schlieBlich Delirien).

Rachicentese (22. Juli). Es wurden ungefähr $20 \mathrm{ccm}$ gleichmäßig trüber Huassigkeit entleert, deren Bodensatz gesammelt und zu Kulturen verwendet wurde.

Am 24. Juli machten wir eine zweite Punktur, wobei nngefähr $25 \mathrm{ccm}$ einer Flüssigkeit gewonnen wurden, die concentrierter und trüber war als die bei der ersten Operation entleerte.

Am 28. Juli starb die Kranke in comatösem Zustande unter meningitischen Erscheinungen, die immer deutlicher sich kundgaben.

Nekroskopie (29. Juli 1904 ).

Skeletbau regelmäßig. Am knöchernen Schädel war eine Continuitătstrennung vorhanden im hinteren Telle der Sutura sagittalis, in der Ausdehnung von ungefäbr $3 \mathrm{~cm}$. Die Dura mater adhärierte an den Rändern der Continuitatstrennung, welche im Knochen sich vorfand, an der inneren Knochentafel, und war entsprechend der Größe jener Continuitätstrennung mit Granulationen, Pseudomembranen und Eiter bedeckt. Das Lumen des Sinus longitudinalis war frei im vorderen Teile bis in die Nähe der Läsion; von hier an aber enthielt derselbe einen eitrigen Thrombus bis zum Confluens sinum, und in einer gewissen Ausdehnung fehlte die außere Wand des Sinus; der Thrombus erstreckte sich auf den ganzen rechten lateralen Sinus und ungefähr auf die Hälfte des linken lateralen.

Die Sinus petrosi und die anderen Sinus der Dura mater beider Seiten waren normal, ebenso auch die beiden Venae jugulares. Im oberen Teile des rechtsseitigen Sulcus sigmoideus, und zwar in der Nähe des von ihm gebildeten Knies befand sich ein ovaler Defekt, mit dem gröBten Durchmesser von $11 / 2 \mathrm{~cm}$ in der Richtung des Sulcus, durch welche hindurch die Sonde in einige vereiterte Zellen des Warzenfortsatzes eindrang. Die Pachymeningitis der Dura des Sinus entsprach zam Teil den genanatem cariösen Knochendefekte.

Eitrige diffuse Leptomeningitis. Die anderen Organe waren gesund, abgesehen von pleuritischen Adbasionen rechts, namentlich entsprechend dem oberen Lappen der Lunge

Untersucbung des rechten Schläfenbeins.

Am Sulcus sigmoideus die erwähnten Lãsionen. Trommelfell, Paukenböble und anch die Vestibularwand derselben normal, das Antrum gleichfalls gesund und communiciert nicht mit denjenigen hinteren Zellen des Warzenfortsatzes, welche dem erodierten Telle des Sinus anliegen.

Es ist in unserem Falle die eiterige Thrombose des reohtsseitigen Sulcus sigmoideus, die consecutiv nach einer akuten 
Mastoiditis mit Erosion des Suleus sigmoideus aufgetreten ist und die Thrombose des hinteren Abschnittes des Sinus longitudinalis super. verursacht hat, intra vitam vollständig latent geblieben. Nur die Thrombose des Sinus longitudinalis hatte sich manifestiert, und zwar durch die Scheitelschwellung, die charakteristisch ist, weil sie von einem in dieser Region konstanten anatomisehen Factum, d. h. von der Anwesenheit eines Emissarium Santorini abbängig ist.

Analog unserem Falle, obwohl von demselben in vielen Beziehungen abweichend, ist eine von De Carli (II. Fall, Atti della Clinica otojatrica di Roma 1904, p. 18) gemachte Beobachtung.

37 Jahre alter Mann. Vor 3 Jabren schwere Malariainfektion. Links seit ungefähr 2 Jahren eiterige Otitis. Kopfschmerz, Parese des Facialis rechts, Entzündung der Papille auf beiden Seiten. Bei der Operation traf man auf der linken Seite einen extraduralen Abszeß um den Sinus herum. Der thrombosierte Sinus lateralis wurde eröffnet und tamponiert. Am 3. Tage wieder intensives Kopfweh, und es trat Schwellung am Scheitel anf, von der Größe einer $\mathrm{NuB}$, fluktuierend, an der Basis von infiltrierten Gewebe umgeben. Abends mäßiges Fieber $(38 \%$. Später: Parese im unteren Teile des Gesichtes links, vollständige Monoplegie der linken oberen, und Parese der unteren Extremität derselben Seite, die als Kompressionserscheinungen aufgefaßt worden sind, weil sie am nächsten lage nicht mehr vorhanden waren. Bei Incision der Schwellung trat blof reichliches Blut aus einem Emissarium Santorini hervor, und es multe desbalb tamponiert werden (Hämatom oder Venendilatation?). Es war weder Schwellung des Gesichtes, noch Turgescenz der Venen vorhanden. Bei der Autopsie fand man phlebitische Thrombose des linksseitigen Sinus lateralis, die sich auf den Sinus longitudinalis sup. (in nicht genan vom Verfasser bestimmter Ausdehnung) fortsetzte. Eiterige Leptomeningitis, Die Parese der Extremitaten dürte durch das Bestehen eines Eiterherdes in der Gegend der Circonvolutio pararolandica auf der rechten Seite erklärt werden.

Der Sitz des Hämatoms im Falle von De Carli war identisch mit dem des Abszesses in unserer Beobachtung und entsprach dem Emissarium Santorini, das durch das Foramen parietale austritt.

Das Auftreten einer Sohwellung an dieser Gegend mub also als charakteristisch fïr eine Thrombose des Sinus longitudinalis superior angesehen werden. Der Schwellung (einfache Venendilatation, Hämatom oder AbszeB) kann sich eine Erweiterung der Venen (Lermoyezsches Symptom), ferner Ödem des behaarten Kopfes, der Stirn und der Lider hinzugesellen.

Weitere Beobachtungen werden dartuber aufklären, warum diese Symptome bloß in gewissen Fällen beobachtet werden. Vielleicht hängt dies von der Variabilität der Beziehungen $a b$, welche in den einzelnen Fällen zwischen der venösen Cirkulation 
250 XIII. GRADENIGO, Über e. charakt. Symptom d. eitrig. Thrombose usw.

des behaarten Kopfes und dem Blutzuflusse zum Sinus longitudinalis besteht. Möglich ist auch, daß die Seltenheit des Vorkommens jener Symptome bloß eine scheinbare und nur dem Umstande zuzuschreiben ist, daß man bis jetzt derselben nicht in methodischer Weise Aufmerksamkeit sehenkte.

Jedenfalls muß ein jedes neue Kennzeichen, das zur Diagnose beitragen und dem Operateur bei den endokraniellen Komplikationen der Ohrentziundungen zu Gute kommen kann, in ernster Weise berücksichtigt werden.

Unter 12 Fällen von eiteriger Thrombose des Sinus longitudinalis superior, welche in dieser Arbeit citiert werden, war $10 \mathrm{mal}$ der Sinus lateralis rechts erkrankt. Diese augenfällige Prävalenz der Erkrankung dés Sinus auf der rechten Seite hängt ohne Zweifel von dem bekannten Umstande $a b$, daß der Sinus longitudinalis superior oft direkt in den rechten Sinus lateralis ausmündet. Hingegen scheint für die Entstehung der gedachten, selten auftretenden Komplikation der Umstand, ob die eiterige Mittelohrentzündung, welche ja die Ursache der Thrombose ist, eine akute oder chronische ist, nicht von Wichtigkeit zu sein. In den angeführten Fällen war die Ohrerkrankung $7 \mathrm{mal}$ ehronisch, 5 mal hatte sie einen akuten oder subakuten Verlauf. In fast allen trat der Tod infolge einer diffusen eiterigen Leptomeningitis ein. 